\title{
Treatment of recurrent gastrointestinal haemorrhage in a patient with von Willebrand's disease with administration of octreotide LAR and propranolol
}

\author{
Napoleon Krikis, Konstantinos Tziomalos, Vasilios Perifanis, Sofia Vakalopoulou, \\ Asterios Karagiannis, Vasilia Garypidou, Fedon Harsoulis
}

Second Propedeutic Department of Internal Medicine, Aristotelian University of Thessaloniki, Ippokration General Hospital, Thessaloniki, Greece

\begin{abstract}
We report a patient with von Willebrand' s disease who had had recurrent and life-threatening bleeding from the gastrointestinal tract. Despite extensive investigation, no apparent cause of haemorrhage was identified. He was successfully treated with combined administration of octreotide LAR (long-acting release) and propranolol. This is the first report on the successful use of octreotide LAR in a patient with von Willebrand' $s$ disease.
\end{abstract}

Keywords: Gastrointestinal haemorrhage, Von Willebrand's disease, Octreotide LAR

\section{INTRODUCTION}

Von Willebrand's disease (vWD) is the most common inherited bleeding disorder. It is caused by quantitative deficiency or qualitative abnormalities of von Willebrand factor (vWF). It is usually an autosomal dominant disease and less frequently an autosomal recessive one, although acquired forms have also been reported. vWD has been classified into three types: Type I, accounting for $70-80 \%$ of patients, represents a quantitative deficiency of vWF; Type II, accounting for $13-18 \%$ of patients, is characterized by several qualitative abnormalities of WF; and Type III, ac-

Address correspondence and requests for reprints to: Konstantinos Tziomalos, 63 Solonos Str., Thessaloniki, 542 48, Phone number: 2310 823487, Fax number: 2310992 834, e-mail address: ktziomalos@yahoo.com

Received 08-11-03, Revised 11-12-03, Accepted 20-12-03 counting for $1-3 \%$ of patients, is characterized by undetectable levels of vWF. Clinical presentation is variable, depending on the type. In types I and II, manifestations include bleeding from mucosal surfaces (epistaxis, bleeding from the gastrointestinal tract and menstrual bleeding), and bleeding with dental extractions or surgical procedures, whereas in type III bleeding mimics the findings seen in hemophilia, including joint bleeding. Diagnosis of vWD should be considered in patients with a personal and family history of bleeding episodes and is established with the following laboratory findings: a) prolonged bleeding time, b) moderately prolonged activated partial thromboplastin time, c) absence of ristocetin-induced platelet aggregation, d) decrease of factor VIII, and e) decrease of vWF antigen and vWF activity ${ }^{1,2}$.

Treatment of patients with vWD depends on the bleeding manifestations and disease type. Until re- 
cently, treatment of bleeding from the gastrointestinal tract in patients with vWD included administration of tranexamic acid, red cell transfusion, intravenous clotting factor products such as fresh frozen plasma and cryoprecipitate (which contains 5-10 times more vWF and FVIII than fresh frozen plasma), and purified $\mathrm{vWF}$ concentrates or preparations that contain $\mathrm{vWF}$ and $\mathrm{FVIII}^{1-3}$. Desmopressin, which promotes the release of vWF from endothelial cell storage sites in mild forms of vWD, has also been used ${ }^{4}$, as well as beta-blocking drugs, which cause a reduction in portal pressure ${ }^{5}$, hormonal therapy with estrogen with or without progesterone ${ }^{2}$, immunoglobulin in acquired forms of $\mathrm{vWD}^{2}$ and surgical resection of lesions that have been clearly identified as the source of bleeding $^{1,2}$.

We report a patient with vWD who has had recurrent and life-threatening bleeding from the gastrointestinal tract, in whom, despite an extensive investigation, no apparent cause of haemorrhage was identified. He was successfully treated with combined administration of octreotide LAR (long active released) and propranolol. This is the first report on the use of octreotide LAR in a patient with vWD.

\section{CASE REPORT}

A 55-year old man presented to our Department because of recurring episodes of melaena, which had first appeared five years previously. During the investigation of his bleeding diathesis, he was found to have type I vWD. He had a history of epistaxis during his childhood. His niece was also diagnosed with type I vWD, whereas his father, brother and grandmother suffered from bleeding diathesis but no specific investigation had been undertaken. Laboratory investigation was compatible with the diagnosis of vWD, with prolonged bleeding time (15 min, normal range 3-8 min), moderate prolongation of activated partial thromboplastin time (41 sec, normal range 20-30 sec), mild deficiency of FVIII (42\%, normal range 50$150 \%$ ), complete absence of ristocetin-induced platelet aggregation, moderate decrease of vWF $(29 \%$, normal range 50-160\%) and a moderate decrease of vWF antigen (33\%, normal range 50-160\%). The diagnosis of type I vWD was based on the findings of prolonged bleeding time, moderate prolongation of activated partial thromboplastin time, as well as moderate decrease of $\mathrm{vWF}$, moderate decrease of $\mathrm{vWF}$ antigen and mild deficiency of FVIII. Platelet count $(300,000 / \mu \mathrm{L})$ and prothrombin time $(12 \mathrm{sec})$ were within normal limits and an extensive laboratory investigation excluded the presence of concomitant disorders.

During the past 17 months, the patient had been admitted 14 times for recurrent episodes of melaena with an overall hospitalized time of 98 days and consequent long sick leaves from his job. On two occasions, hemoglobin concentration on admission was 6 $\mathrm{g} / \mathrm{dL}$. He required 40 red cell transfusions and 22,000 IU of purified vWF. During this period, upper endoscopy was performed five times, small bowel series radiography twice and colonoscopy three times; computed tomography of the abdomen, radionuclide scanning with $99 \mathrm{mTc}$ pertechnate-labeled autologous red blood cells, angiography of the superior mesenteric artery and exploratory surgery with intraoperative enteroscopy were also performed, but the source of bleeding could not be localized. He had received intranasal desmopressin for three months with no effect on the frequency of bleeding. He was subsequently treated with octreotide LAR 20mg (Sandostatin LAR, Novartis, Athens, Greece) IM once a month, along with propranolol $20 \mathrm{mg}$ per os three times per day. With this therapeutic regimen the bleeding stopped completely, the hemoglobin values stabilized at normal levels $(13.2 \mathrm{~g} / \mathrm{dL})$ and no treatment-related side effects were observed. During a follow-up period of eight months, bleeding did not recur and the patient has returned to his work. Repeated evaluation of vWD revealed that $\mathrm{vWF}$ levels did not rise $(28 \%)$, ristocetin-induced platelet aggregation remained absent and activated partial thromboplastin time and bleeding time prolongations remained unchanged.

\section{DISCUSSION}

We have shown that recurrent bleeding from the gastrointestinal tract can be successfully managed with the combined administration of octreotide LAR and propranolol. We must point out that no apparent source of bleeding could be determined and the haemorrhage could not be controlled with appropriate treatment applied for many months (red cell transfusions, administration of desmopressin and vWF), while surgical treatment was not applicable since the source of bleeding could not be located. 
Octreotide is mainly used in acromegaly and in gastrointestinal and pancreatic tumors ${ }^{6-8}$. Nevertheless, octreotide has been proved to be effective in controlling bleeding from the gastrointestinal tract due to angiodysplasia and variceal bleeding ${ }^{6,9-13}$. It is postulated that it exerts its actions through a reduction in splachnic and portal blood flow ${ }^{6}$. Octreotide LAR, compared to conventional octreotide, has the advantage that it is administered once monthly and does not require hospitalization while exhibiting a similar efficacy and safety profile ${ }^{14,15}$.

Only one report on the effectiveness of octreotide therapy in two patients with vWD was found in the literature. In these two patients angiodysplasias (located in the stomach, duodenum and small bowel) were detected ${ }^{16}$. In our patient, no angiodysplasias were found despite an extensive work-up. Wireless capsule endoscopy is currently the gold standard for the diagnosis of small intestine angiodysplasias ${ }^{17}$; unfortunately, this technique is not available in our Hospital. Therefore, the possibility of an unrecognized angiodysplasia, which could have been the source of gastrointestinal bleeding in our patient, still exists. Finally, in one of the previously reported patients ${ }^{16}$, vWF was increased after the administration of octreotide. In our patient octreotide did not cause any increase in the synthesis or release of vWF.

Undoubtedly, more trials are required in order to clarify the mechanism of action of octreotide in this setting. Whatever the mechanism involved, combined administration of octreotide and propranolol appears to be an attractive alternative treatment in these rare and life-threatening conditions, when other therapeutic modalities have failed.

\section{REFERENCES}

1. Rizza C, 1997 Von Willebrand's disease In: Rizza C, Lowe G (eds) Haemophilia and other inherited bleeding disorders, WB Saunders Company Ltd, London; pp, 103-109.

2. Mannuci PM, 2001 How I treat patients with von Wille- brand disease. Blood 97: 1915-1919.

3. Mannuci PM, 1998 Hemostatic drugs. N Engl J Med 339: 245-253.

4. Kaufmann JE, Oksche A, Wollheim CB, Gunther G, Rosenthal W, Vischer UM, 2000 Vasopressin-induced von Willebrand factor secretion from endothelial cells involves V2 receptors and cAMP. J Clin Invest 106: 107-116.

5. Schwartz J, Rozenfleld V, Habot B, 1992 Cessation of recurrent bleeding from gastrointestinal angiodysplasia after beta-blocker treatment in a patient with hypertrophic subaortic stenosis-a case history. Angiology 3: 244-248.

6. Lamberts S, van der Lely AJ, de Herder WW, Hofland LJ, 1996 Octreotide. N Eng J Med 25: 246-254.

7. Freda PU, 2002 Somatostatin analogues in acromegaly. J Clin Endocrinol Metab 87: 3013-3018.

8. Gillis JC, Noble S, Goa KL, 1997 Octreotide long-acting release (LAR). A review of its pharmacological properties and therapeutic use in the management of acromegaly. Drugs 4: 681-699.

9. Orsi P, Guatti-Zuliani C, Okolicsanyi L, 2001 Long-acting octreotide is effective in controlling rebleeding angiodysplasia of the gastrointestinal tract. Dig Liver Dis 33: 330-334.

10. Andersen MR, Aaseby J, 1996 Somatostatin in the treatment of gastrointestinal bleeding caused by angiodysplasia. Scand J Gastroenterol 31: 1037-1039.

11. Rossini FP, Arrigoni A, Pennazio M, 1993 Octreotide in the treatment of bleeding due to angiodysplasia of the small intestine. Am J Gastroenterol 9: 1424-1427.

12. Lewis BS, 1999 Medical and hormonal therapy in occult gastrointestinal bleeding. Semin Gastrointest Dis 2: 7177.

13. Nardone G, Rocco A, Balzano T, Budillon G, 1999 The efficacy of octreotide therapy in chronic bleeding due to vascular abnormalities of the gastrointestinal tract. Aliment Pharmacol Ther 11: 1429-1436.

14. Bajetta E, Bichisao E, Artale S, et al, 2000 New clinical trials for the treatment of neuroendocrine tumor. Q J Nucl Med 1: 96-101.

15. Anthony LB, 1999 Long-acting formulations somatostatin analogues. Ital J Gastroenterol Hepatol 31: 216-218.

16. Bowers M, McNulty O, Mayne E, 2000 Octreotide in the treatment of gastrointestinal bleeding caused by angiodysplasia in two patients with von Willebrand's disease. Br J Haematol 108: 524-552.

17. Mylonaki M, Fritscher-Ravens A, Swain P, 2003 Wireless capsule endoscopy: a comparison with push enteroscopy in patients with gastroscopy and colonoscopy negative gastrointestinal bleeding. Gut 52: 1122-1126. 